\title{
La diversité territoriale des régimes français de financement public des cultes
}

\section{Jean-Marie Woehrling}

\section{OpenEdition}

\section{Journals}

Édition électronique

URL : http://journals.openedition.org/rdr/1041

DOI : $10.4000 /$ rdr. 1041

ISSN : 2534-7462

\section{Éditeur}

Presses universitaires de Strasbourg

Édition imprimée

Date de publication : 10 mai 2016

Pagination : $67-84$

ISBN : 978-2-86820-954-2

ISSN : 2493-8637

\section{Référence électronique}

Jean-Marie Woehrling, «La diversité territoriale des régimes français de financement public des cultes », Revue du droit des religions [En ligne], 1 | 2016, mis en ligne le 12 février 2020, consulté le 19 novembre 2020. URL : http://journals.openedition.org/rdr/1041 ; DOI : https://doi.org/10.4000/rdr. 1041

La revue du droit des religions est mise à disposition selon les termes de la Creative Commons Attribution - Pas d'Utilisation Commerciale 4.0 International - CC BY-NC 4.0. 


\section{LA DIVERSITÉ TERRITORIALE DES RÉGIMES FRANÇAIS DE FINANCEMENT PUBLIC DES CULTES}

\section{Jean-Marie WOEHRLING}

Président de l'Institut du droit local alsacien-mosellan

\section{RÉSUMÉ}

La mise en œuvre d'un droit différencié selon les territoires, qui constitue une forme d'adaptation de l'organisation juridique à la diversité historique, culturelle ou géographique des situations locales, trouve une application remarquable en droit français des religions en ce qui concerne les modalités de soutien financier public aux activités religieuses. Elle s'exprime de deux manières : la non-application de la loi du 9 décembre 1905 dans certains territoires ; l'existence de statuts particuliers pour certains cultes dans certaines régions. Cette diversité de régimes juridiques en matière religieuse est-elle compatible avec les principes constitutionnels? La jurisprudence du Conseil constitutionnel garantit la sauvegarde des régimes historiques au regard de ces principes, mais restreint toute évolution qui ne va pas dans le sens d'un rapprochement avec le droit commun.

\section{ABSTRACT}

The territorial differentiation of French law, which is a form of adaptation of the legal regime to various historical, cultural or geographical local situations, fully applies to the public funding of religious activities. It is expressed in two ways: the non-application of the 1905 Act on the separation in some territories; the existence of special status accorded to certain denominations in some areas. Is this diversity of legal systems in religious matters compatible with the constitutional principles? The jurisprudence of the Constitutional Council ensures the continuation of historical regimes in light of these principles, but restricts any development departing from the common law. 
T e droit local alsacien-mosellan a mis en lumière un phénomène de

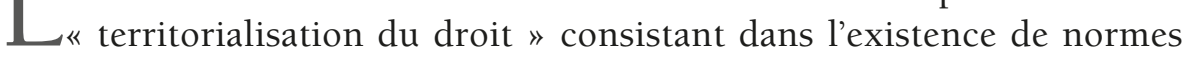
particulières à un territoire au sein de l'État unitaire ${ }^{1}$. Le droit local alsacien-mosellan constitue une illustration de ce type de situation juridique, qui concerne aussi les collectivités d'outre-mer ainsi que des règles territoriales particulières à certaines régions métropolitaines (Corse, région parisienne, etc.).

Cette variation des règles juridiques en fonction des territoires concerne notamment le régime de financement des cultes. Bien qu'unitaire, la République admet que cette matière puisse relever de règles différentes pour tenir compte de l'histoire et des situations culturelles ou géographiques particulières. Il s'agit là d'un aménagement remarquable et souvent méconnu du droit français des religions. Il existe des situations profondément différentes dans l'organisation du financement des cultes (1) qui pourraient apparaître comme une source d'inspiration pour une adaptation géographique des relations États-Églises (2), mais dont le développement semble difficilement compatible avec les principes dégagés par le Conseil constitutionnel (3).

\section{ORIGINALITÉ DES MODALITÉS DE FINANCEMENT PUBLIC DES CULTES PROPRES À CERTAINS TERRITOIRES}

Ce sont plusieurs territoires qui bénéficient de régimes spécifiques en matière de financement des cultes. Dans le cas de l'Alsace et de la Moselle, le régime particulier résulte de l'histoire. Pour les autres cas, c'est-à-dire certaines régions d'outre-mer, c'est plutôt la géographie - c'est-à-dire l'éloignement, mais aussi la spécificité culturelle -, qui explique l'existence d'une législation particulière.

Les spécificités territoriales en matière de droit des religions sont assez diverses. On se bornera ici aux seules règles relatives au financement des activités religieuses. À cet égard, les spécificités juridiques peuvent être regroupées selon trois éléments : la non-application de la loi de 1905, l'existence de statuts particuliers pour certains cultes et enfin un certain nombre de dispositions accessoires.

1. Woenrling J.-M. (dir.), Du droit local à la territorialisation du droit : perspectives et limites, Strasbourg, Institut du droit local alsacien-mosellan, 2014. 


\subsection{LA NON-APPLICATION DE LA LOI DU 9 DÉCEMBRE 1905 SUR LA SÉPARATION DE L'ÉTAT ET DES ÉGLISES}

L'ensemble des régimes territoriaux spécifiques en matière de financement des cultes est caractérisé par la non-introduction dans ces territoires de la loi du 9 décembre 1905, notamment de son article 2 selon lequel « la République ne salarie ni ne subventionne aucun culte».

En Alsace et Moselle, la législation applicable à ces territoires au moment de leur retour à la France est restée en vigueur sous réserve de l'introduction progressive de la législation française. La loi de 1905 n'a pas été introduite en raison de l'hostilité des populations locales à la remise en cause du régime des cultes reconnus maintenus en vigueur pendant la période allemande.

Pour les régions d'outre-mer, l'article 43 de la loi de 1905 a prévu que des décrets en Conseil d'État détermineront les conditions dans lesquelles la loi sera applicable aux colonies (aujourd'hui les départements, régions ou collectivités d'outre-mer, ainsi que la Nouvelle-Calédonie, régis respectivement par les articles 72 à 76 de la Constitution). En application de ces dispositions, un décret du 6 février 1911 a étendu la loi de 1905 aux îles de la Réunion, de la Guadeloupe et de la Martinique ${ }^{2}$. En revanche, la Guyane, la NouvelleCalédonie, la Polynésie française ${ }^{3}$, Mayotte, Wallis et Futuna, Saint-Pierre et Miquelon, les Terres australes et antarctiques françaises ${ }^{4}$ ne sont pas soumises à cette $\operatorname{loi}^{5}$.

Il convient de préciser les effets de cette non-application. À défaut d'entrée en vigueur de la loi du 9 décembre 1905 dans les territoires considérés, il n'existe aucune limitation spécifique au financement public des cultes. En

2. Un décret de 1907 avait aussi étendu la loi de 1905 à l'Algérie et un décret de 1911 à Madagascar ; mais la mise en œuvre effective de cette loi dans ces colonies est restée très partielle.

3. Un texte spécial applicable à la Polynésie française, le décret du 5 juillet 1927 portant statut du culte protestant, prévoit en son article 7 le financement public des aumôneries dans les prisons, hôpitaux et armées. Mais finalement cette disposition se recoupe avec les règles applicables en métropole. Il devait être supprimé par l'ordonnance n 2009-536 du 14 mai 2009 portant diverses dispositions d'adaptation du droit outre-mer. Le Parlement a cependant décidé de ne pas ratifier l'article 11 de l'ordonnance susmentionnée en tant qu'il prévoit l'abrogation de ce décret de 1927.

4. Dans les Terres australes et antarctiques françaises, les édifices du culte appartiennent à l'État, qui en assure l'entretien. La situation n'est pas très différente du droit commun.

5. Regnault J.-M., « Présentation: application et non-application outre-mer de la loi de séparation de $1905 »$, Outre-mers, n 348-349, 2005, p. 5-7. 
effet, selon la jurisprudence tant du Conseil d'État ${ }^{6}$ que du Conseil constitutionnel ${ }^{7}$, l'article 2 de cette loi selon lequel la République ne subventionne aucun culte n'a pas de valeur constitutionnelle. Cette liberté de financement vaut aussi bien pour l'État que pour les collectivités territoriales et concerne les soutiens financiers directs comme les avantages indirects (mise à disposition de terrains, locaux ou autres équipements).

L'attribution de soutiens financiers par l'État ou les collectivités territoriales est par exemple possible au bénéfice d'organisations relevant du culte musulman ${ }^{8}$. Un certain nombre de mosquées ont d'ailleurs bénéficié de subventions de collectivités locales. Toutefois, si aucune règle législative spéciale ne limite l'attribution de soutiens financiers aux activités religieuses, un certain nombre de règles générales restent applicables en la matière.

En premier lieu, tout financement public est subordonné à l'existence d'un intérêt public justifiant son attribution. Cette règle s'applique sans discussion pour les collectivités territoriales, ainsi que l'a rappelé le Conseil d'État, même quand la loi de 1905 n'est pas applicable ${ }^{9}$. Une interprétation restrictive de l'intérêt général justifiant l'octroi d'un soutien financier peut conduire à une « introduction larvée » du principe de non-subventionnement des cultes en Alsace-Moselle, comme peuvent l'illustrer certaines espèces ${ }^{10}$. Cette exigence d'un intérêt public vaut aussi pour les aides attribuées par l'État.

En deuxième lieu, l'aide financière à des activités religieuses ne doit pas être discriminatoire. En pratique cependant, les différents cultes ne bénéficient pas de la même attention des collectivités : compte tenu du souci de favoriser l'intégration des familles issues de l'immigration, l'aide apportée au culte musulman est significative, alors qu'il n'y a guère de soutien aux mouvements évangéliques.

Enfin, dans le cas d'un soutien émanant de collectivités locales, celui-ci doit être justifié par un intérêt local. De plus, avec la loi n 2015-991 du 7 août 2015, les régions et les départements ont perdu la clause générale de

6. CE, 16 mars 2005, n 265560, Ministre de l'Outre-mer, AJDA 2005, note Durand-Prinborgne Cl., p. 1463 ; Revue du droit local, n 44, 2005, note Woenrling J.-M., p. 19.

7. Cons. const., déc. 21 févr. 2013, n² 2012-297 QPC, Association pour la promotion et l'extension de la laïcité (APPEL), AJDA 2013, note FOREY E., p. 1008.

8. Ce soutien n'implique nullement, comme on l'entend dire parfois, une hypothétique « extension» du statut des « cultes reconnus » alsacien-mosellan à l'islam.

9. CE, 16 mars 2005, précit.

10. CAA Nancy, 6 mars 2008, n 07NC00083, Commune de Soultz: la subvention versée à un groupe de jeunes actifs dans la commune pour effectuer un voyage est déclarée dépourvue d'intérêt général car le voyage comporte une visite au Vatican. 
compétence, ce qui les prive d'une capacité de soutien aux activités religieuses en raison de leur caractère d'intérêt régional ou départemental, sauf à mettre en avant un éventuel intérêt culturel.

Dans les territoires relevant de l'outre-mer, le décret « Mandel» du 16 janvier 1939 encadre l'activité cultuelle du point de vue civil : ce décret donne la personnalité juridique aux « missions religieuses », mais reste muet sur la question des soutiens financiers publics. Les articles 4 et 5 de ce décret comportent cependant des dispositions fiscales et les articles 8 et suivants règlent des questions relatives aux libéralités faites à des organisations religieuses ${ }^{11}$.

\subsection{L'EXISTENCE DE « STATUTS PARTICULIERS » POUR CERTAINS CULTES}

Plusieurs territoires sont caractérisés par le fait que certains cultes bénéficient de statuts législatifs particuliers qui concernent notamment leur financement. Ces statuts particuliers sont caractéristiques pour l'Alsace et la Moselle, mais il existe aussi un tel statut particulier pour la Guyane et Saint-Pierre et Miquelon. À Mayotte, la départementalisation a fait disparaître, du moins en droit, les spécificités en matière de financement des cultes ${ }^{12}$.

\subsubsection{Les statuts particuliers reconnus en matière de financement public à certains cultes en Alsace et Moselle}

En vertu de textes spéciaux, certains cultes (le culte catholique, deux cultes protestants et le culte israélite) relèvent de statuts particuliers comportant des

11. V. infra. 1.3.

12. À Mayotte, la loi du 9 décembre 1905 n’a pas été introduite, bien que l'île représentait à l'époque une dépendance de Madagascar (le décret du 11 mars 1913 pris sur le fondement de l'article 43 de la loi du 9 décembre 1905 et relatif à l'introduction de cette loi à Madagascar avait renvoyé à des textes ultérieurs, jamais introduits, l'application de cette loi à Mayotte). En vertu d'un arrêté du gouverneur de Madagascar du 10 mars 1939, c'est le décret du 11 janvier 1939 qui s'applique à cette île, sauf les articles 2 et 8 de celui-ci résultant d'un décret ultérieur. Selon un avis du Conseil d'État du 20 mai 2010, en application de l'article LO6113-1 du Code général des collectivités territoriales, les dispositions qui prévalaient à Mayotte dans le domaine cultuel avant le passage au " régime de l'identité » (c'est-à-dire son accession le 31 mars 2011 au statut de département d'outre-mer régi par l'article 73 de la Constitution) restent applicables. Depuis l'ordonnance ${ }^{\circ} 2010-590 \mathrm{du}$ 3 juin 2010, les cadis, s'ils demeurent ministres du culte musulman n'ont plus de fonctions juridictionnelles civiles et ne sont plus rémunérés à ce titre. Si les dispositions de l'article L. 1311-2 du Code général des collectivités territoriales relatives au bail emphytéotique ne sont pas étendues à Mayotte, par contre l'article L. 451-1 du Code rural et de la pêche maritime permet des baux emphytéotiques sur les dépendances du domaine privé des collectivités territoriales pour l'édification de bâtiments cultuels. 
contraintes spécifiques (un droit de regard des pouvoirs publics) en échange de certains avantages. Parmi ces derniers figure un soutien financier qui comporte un double aspect. Tout d'abord, si l'entretien des édifices cultuels et logements des ministres du culte, qui constituent des ouvrages publics, relève à titre principal des fabriques et autres établissements publics du culte, les communes ont l'obligation de subvenir aux besoins de ces établissements cultuels dans le cas où ceux-ci seraient en situation d'insuffisance de revenus ${ }^{13}$. Dans la pratique, les établissements publics du culte sont en mesure de subvenir aux frais ordinaires du culte, mais les collectivités locales doivent intervenir pour des dépenses plus lourdes, telles que des grosses réparations. Par ailleurs, les communes peuvent mettre un logement à disposition des pasteurs, curés et desservants, rabbins et grands rabbins légalement institués. À défaut, le paiement d'une indemnité compensatrice de logement incombe à ces collectivités ${ }^{14}$.

Les ministres des quatre cultes statutaires occupant des postes prévus au budget de l'État sont rémunérés par le ministère de l'Intérieur. Cette prise en charge financière résulte de l'article 14 de la convention de messidor an IX (concordat) pour le culte catholique et de l'article 7 des articles organiques pour les cultes protestants (loi du 18 germinal an $\mathrm{X}$ ), ainsi que d'une loi du 8 février 1831 pour le culte juif. À ces dispositions s'est substituée une loi locale du 15 novembre 1909. Cette loi reprend aussi bien le principe même d'une rémunération pour les ministres du culte protestant que les modalités de cette rémunération. En ce qui concerne le montant des rémunérations, qui relève du pouvoir réglementaire, c'est désormais un décret n 48-1108 du 10 juillet 1948 qui définit un classement et un échelonnement indiciaire des ministres du culte. En vertu de la loi de 1909, le ministère de l'Intérieur rémunère aussi des laïcs exerçant des fonctions administratives au service des autorités religieuses supérieures. Des laïcs exerçant des fonctions pastorales sont dans certains cas placés sur des postes vacants de vicaires ou de desservants. Au total, ce système de rémunération correspond à environ 1400 emplois représentant environ 58 millions d'euros.

\subsubsection{Régime particulier du culte catholique en Guyane}

La Guyane reste caractérisée par l'application de l'ordonnance royale de Charles X du 27 août 1828, ni la loi du 9 décembre 1905, ni le décret du

13. Décret du 9 décembre 1809 ; art. L. 2543-3 CGCT.

14. Art. 92 du décret du 30 décembre 1809 pour le culte catholique; décret du 15 mai 1806 pour le culte protestant ; article 30 loi du 18 juillet 1837 ; CE, avis, 26 avril 1994. 
6 février 1911 déterminant les conditions d'application de cette loi dans un certain nombre de colonies n'ayant été rendus applicables à ce territoire. Par contre s'y applique le décret du 16 janvier 1939 dit « décret Mandel ${ }^{15}$. En vertu de l'article 33 d'une loi du 13 avril 1900 portant fixation du budget et d'un décret d'application du 21 août 1900, les « dépenses de personnel et de matériel nécessaires au culte » font partie des dépenses obligatoires « à la charge de la colonie de Guyane ", c'est à dire aujourd'hui le département de la Guyane. Toutefois, seul le personnel agréé comme ministre culte par le préfet bénéficie de cette prise en charge. Actuellement 27 ministres du culte catholique de Guyane sont rétribués sur le budget départemental comme agents permanents titulaires après avoir reçu l'agrément de l'autorité préfectorale. Le Conseil d'État a confirmé que ces dispositions restaient en vigueur ${ }^{16}$. Les membres du clergé catholique sont ainsi rémunérés sur le budget des emplois départementaux ${ }^{17}$.

Les bâtiments cultuels catholiques construits avant 1939, c'est le cas de la plupart des églises catholiques de Guyane, l'ont été sur des terrains appartenant au département ou aux communes et sont entretenus par ces collectivités. Les constructions postérieures à 1939 appartiennent à la mission catholique de Guyane. Dans les deux cas, en vertu de la loi sus-rappelée de 1900, les bâtiments cultuels sont à la charge du département. Les missions religieuses bénéficient pour leurs biens de l'exonération de la taxe foncière ${ }^{18}$.

\subsubsection{Saint-Pierre et Miquelon}

À Saint-Pierre et Miquelon, depuis le ralliement de l'archipel à la France libre $^{19}$, les prêtres catholiques sont rémunérés sur fonds publics de la collectivité territoriale sans que cette pratique ait une base légale précise. Les églises appartiennent aux communes qui pourvoient à leur entretien.

15. Décret ayant été rendu applicable à la Guyane par un arrêté du gouverneur du 26 août 1939.

16. CE, 9 oct. 1981, Beherec.

17. Coût annuel : environ 800000 euros de fonds publics, soit selon l'évêque, mois de $1 \%$ du budget de la collectivité qui compte plus de 2000 agents.

18. CE, 19 juin 2006, Association "La mission du plein évangile - La porte ouverte chrétienne "

19. Poulat É., « La Guyane et son régime religieux dans le système français », in BAUbÉROT J. et Regnault J.-M. (dir.), Relations, Églises et autorités outre-mer : de 1945 à nos jours, Paris, Les Indes savantes, 2007, p. 96. 


\subsection{DISPOSITIONS ACCESSOIRES À INCIDENCE FINANCIÈRE}

On peut distinguer divers aspects :

- Aspects fiscaux : le décret du 16 janvier 1939 portant institution aux colonies de conseils d'administration des missions religieuses (décret Mandel) prévoit que les biens immeubles des missions religieuses servant à l'exercice du culte, à un usage scolaire et constituant des établissements d'assistance médicale ou d'assistance sociale échappent à la législation locale. Selon le Conseil d'État, ces dispositions assurent aux missions un régime fiscal « au moins aussi favorable » que celui prévu pour les associations de la loi de $1905^{20}$.

En Alsace-Moselle, il existe des dispositions spécifiques concernant le non-assujettissement à la taxe foncière des bâtiments affectés au culte appartenant à des associations ${ }^{21}$. Mais ces dispositions visent à faire bénéficier les associations de droit local ayant pour objet exclusif l'exercice d'un « culte non reconnu » des mêmes exonérations que celles dont bénéficient les associations de la loi de 1905 pour les bâtiments leur appartenant et affectés à l'exercice du culte. Les édifices du culte, évêchés, presbytères, séminaires, logements de ministres du culte sont en effet exonérés de la taxe foncière ${ }^{22}$.

- Dons et legs : en Alsace-Moselle, toutes les associations de droit local inscrites au registre des associations ont la pleine capacité juridique et peuvent recevoir des dons et legs, sous réserve bien sûr qu'il n'y ait pas opposition de l'autorité administrative ${ }^{23}$. Selon une réponse ministérielle, les dons et legs faits à des établissements du culte ou à des associations ayant une mission cultuelle en Alsace-Moselle bénéficient de l'exonération établie au bénéfice des établissements charitables ${ }^{24}$.

Dans les territoires qui relèvent du décret Mandel, les dispositions sont moins favorables. Aux termes des articles 8 à 10, les missions religieuses

20. CE, 19 juin 2006, précit. Le Conseil d’État en déduit que l’association requérante, association ordinaire de la loi de 1901, n'est pas fondée à se plaindre de ne pas bénéficier d'une exonération fiscale équivalente à celle dont bénéficient les associations cultuelles de la loi de 1905. Faut-il comprendre que l'association requérante n'est pas discriminée, car elle aurait pu se transformer en mission religieuse pour bénéficier de l'exonération fiscale recherchée?

21. CGI, art. $13824^{\mathrm{e}}$.

22. Code des impôts directs et taxes assimilées applicables en Alsace-Moselle, art 4.

23. C. civ., art. 910, modifié par l'ord. n 2005-856 du 28 juillet 2005.

24. Rép. min. no 40599 : JOAN Q 23 avril 1984, p. 1941 et rép. min. n 612, JOAN Q 6 oct. 1986, p. 3518. V. aussi Doct. adm. DGI, 7 G-261, $\$ 20$ et s., $1^{\text {er }}$ juill. 1987. 
peuvent recevoir, sous réserve de l'autorisation du chef de la colonie, des legs de citoyens français ou de personnes de statut européen ou assimilé, mais non « d'indigènes n'ayant pas la qualité de citoyen français ». De même est soumise à l'autorisation du chef de la colonie, l'acceptation par les missions religieuses de tous dons d'immeubles ou de droits immobiliers et de tous dons en espèces supérieurs à 10000 francs $^{25}$, ou d'effets et objets mobiliers dont la valeur excède cette somme.

\section{SIGNIFICATION DE LA DIVERSITÉ TERRITORIALE EN MATIÈRE DE FINANCEMENT DES CULTES}

L'existence de règles différentes en matière de financement des cultes peut apparaître comme surprenante dans un État qui met l'accent sur l'unité et l'égalité de l'organisation publique. Elle s'explique par le souci de tenir compte de circonstances particulières, qu'elles soient historiques ou géographiques.

Dans le cas de l'Alsace et de la Moselle, les autorités françaises ont accepté de prendre en compte l'expérience historique particulière de ces territoires et de respecter leurs traditions particulières ${ }^{26}$. Si en droit, le maintien du droit local a été considéré comme une simple concession provisoire, au plan politique, on peut parler de la création d'une " tradition républicaine " consistant à respecter la volonté de la population des trois départements de conserver ce régime particulier qui pourra bientôt fêter son $100^{\mathrm{e}}$ anniversaire. Entre temps, si les populations d'Alsace et de Moselle n'ont plus de façon prééminente le souci de préserver la situation des cultes traditionnels de la région, elles restent très fortement attachées au régime local des cultes en tant qu'expression de l'identité régionale. Avec le temps, le système alsacien-mosellan est apparu à nombre d'observateurs comme plus adapté aux attentes actuelles que celui issu de la loi de 1905 (en tant qu'il propose des rapports organisés entre l'État et les cultes les plus importants et en aménageant les rapports de ces derniers au sein de la société).

C'est donc aujourd'hui un ensemble d'éléments qui fondent le régime particulier de financement des cultes d'Alsace et de Moselle : tradition, volonté d'affirmer une personnalité locale, avantages objectifs du système, etc. Même

25. Soit environ 15 euros (Circulaire NOR/4IOCD1121265C du 25 août 2011 sur la réglementation des cultes outre-mer, p. 14).

26. V. Woehrling J.-M., " Le droit local alsacien-mosellan des cultes après les récentes décisions du Conseil constitutionnel », RDP 2013, p. 532. 
si cette législation locale fait l'objet d'un certain nombre de contestations récurrentes, elle continue à bénéficier d'une large adhésion de la population et des élus locaux. Par contre, le caractère ancien de la législation locale impliquerait son évolution afin d'assurer une meilleure adaptation au contexte actuel.

S'agissant de la situation dans les collectivités d'outre-mer, les raisons historiques de la non-introduction de la loi de 1905 ont été incontestablement liées aux relations étroites entre les missions chrétiennes et la présence française dans ces territoires. Dans la période récente, cette motivation a perdu de son importance, mais les pouvoirs publics n'ont pas souhaité revenir sur les situations créées. Selon certains ${ }^{27}$, les considérations budgétaires auraient joué un rôle car l'État peut continuer de recourir aux cultes pour l'assister dans l'exercice des services publics de la santé, de l'éducation et de la solidarité nationale.

Avec la loi du 19 mars 1946 procédant à la départementalisation, la question d'une application de la loi de 1905 s'est posée pour la Guyane. Mais le ministre de l'Intérieur de l'époque, Jules Moch, fait part de ses réticences à l'idée de modifier cette situation « en raison de la pauvreté des habitants de la Guyane et de la nécessité de les soustraire aux influences étrangères que favoriserait un départ des missionnaires catholiques ». La Guyane, explique-t-il en 1948 dans une lettre à son collègue des Finances René Mayer, " a toujours été considérée comme pays de missions parmi les infidèles dont les frais de culte ont été assumés à l'origine par le ministère de la Marine, puis par le ministère des Colonies». Le ministre de l'Intérieur conseille toutefois de "maintenir les pratiques actuelles de la rétribution ecclésiastique par le département de la Guyane et non par le budget de l'État».

Malgré des demandes pressantes des organisations laïques d'introduire la loi de 1905 dans toutes les régions d'outre-mer et de supprimer les régimes cultuels spéciaux, le maintien des aménagements anciens a prévalu. La position des populations locales semble globalement être en faveur du statu quo.

Dans le contexte du renforcement de l'autonomie juridique des collectivités d'outre-mer et, de façon générale, du progrès de l'idée qu'il y a lieu de renforcer la possibilité pour les différents territoires de bénéficier de régimes

27. Demetz A., V « Outre-mer», in Cerf M., Horwitz M. (dir.), Dictionnaire de la laïcité, Paris, A. Colin, 2011, p. 256. 
spécifiques juridiquement adaptés à leurs situations particulières ${ }^{28}$, on aurait pu penser que les questions d'organisation financière des cultes puissent être aménagées de manière spécifique dans le cadre des instances locales. Une demande de ce type a existé au moins en ce qui concerne la NouvelleCalédonie. Mais le pouvoir central n'a pas admis l'interprétation donnée de leur champ de compétence par les autorités locales concernées. En effet, l'ensemble du dispositif juridique institué par le décret précité du 16 janvier 1939 est applicable en Nouvelle-Calédonie. Après l'entrée en vigueur de la loi n ${ }^{\circ} 88-1028$ du 9 novembre 1988 portant dispositions statutaires et préparatoires à l'autodétermination de la Nouvelle-Calédonie en 1998, l'assemblée de la Province Sud, considérant que cette compétence en matière de cultes ne relevait plus de l'État, mais des provinces, avait adopté une délibération $n^{\circ}$ 15-91/APS du 14 mars 1991 adaptant le décret du 16 janvier 1939 pour son application en Province Sud. Par un arrêt rendu par son assemblée du contentieux le 29 avril $1994^{29}$, le Conseil d'État, contredisant l'interprétation soutenue par la Province Sud, a confirmé la compétence de l'État en matière de liberté d'association. Dès lors, la délibération de 1991 précitée ne s'applique pas et le haut-commissaire de la République, qui exerce de nouveau la plénitude de ses compétences en ce domaine, assure l'exécution et l'application des décrets Mandel en Nouvelle-Calédonie.

En Guyane, la loi fait obligation au département de rémunérer les prêtres, mais cette collectivité territoriale n'a pas compétence pour aménager cette obligation légale. Le conseil général de la Guyane a par décision du 14 mai 2014 décidé de supprimer ce financement. Saisi par l'évêque et les 26 prêtres concernés, le juge du tribunal administratif de Guyane a ordonné le 29 décembre 2014 au conseil général de reprendre le versement de ces salaires ${ }^{30}$. La compétence de poursuivre ou de supprimer ce financement relève en effet du législateur. On peut penser que la décision du conseil général ne visait pas tant à obtenir la fin de la rémunération publique des ecclésiastiques catholiques que le transfert de sa charge du département à l'État. Selon la presse, le conseil général compte contester, d'une part, le jugement rendu par

28. Cette idée du respect de la situation géographique particulière se retrouve de manière variable dans les différents statuts des territoires d'outre-mer. Aux termes de l'article 73 de la Constitution, « Dans les départements et régions d'outre-mer, les lois et règlements [...] peuvent faire l'objet d'adaptations tenant aux caractéristiques et contraintes particulières de ces collectivités». L'article 74 dispose que «les collectivités d'outre-mer [...] ont un statut qui tient compte des intérêts propres de chacune d'elles au sein de la République ». Les articles 76 et 77 prévoient de larges transferts de compétence à la Nouvelle-Calédonie.

29. CE, 29 avril 1994, n 119562, Haut-Commissaire de la République en Nouvelle-Calédonie.

30. TA Cayenne, 29 déc. 2014, nº 1400708. 
le tribunal administratif de Cayenne, et d'autre part, intenter une action en justice contre l'État pour lui demander de rembourser à la collectivité départementale les rémunérations versées au culte catholique depuis la départementalisation de 1946. Dans un contexte de polémique croissante, le président du conseil général, Alain Tien-Liong, a pris une "décision de mise à la retraite d'office » de l'évêque.

\section{QUESTIONS CONSTITUTIONNELLES}

La validité constitutionnelle des régimes territoriaux historiques de financement des cultes a été garantie par la jurisprudence. Mais le droit constitutionnel fixe des limites importantes à l'évolution de ces systèmes.

\subsection{LA COMPATIBILITÉ CONSTITUTIONNELLE DES RÉGIMES HISTORIQUES DE FINANCEMENT PUBLIC DES CULTES}

Au moment de l'adoption des textes constitutionnels en 1946, puis en 1958, le constituant n'avait pas l'intention de remettre en cause les régimes particuliers de financement des cultes existant sur certains territoires. Le Conseil constitutionnel en a tiré la conséquence que les dispositions constitutionnelles ne pouvaient être analysées comme ayant remis en cause ces régimes. Cette analyse en termes d'intention du constituant a été critiquée. Elle n'est cependant pas dépourvue de légitimité, car il y a quelque chose de factice dans le fait de découvrir cinquante ans après l'adoption d'un texte constitutionnel que celui-ci avait pour effet d'abroger une législation qui avait cependant continué à s'appliquer. En « découvrant » en 2013 qu'une disposition constitutionnelle de 1946 a abrogé des textes anciens, on donnerait en fait une portée objectivement rétroactive à la remise en cause de ces textes. Le législateur reste cependant compétent pour abroger ces dispositions pour l'avenir.

Quoi qu'il en soit, en vertu de la jurisprudence constitutionnelle, les régimes territoriaux de financement public des cultes sont protégés contre une contestation constitutionnelle. Par contre, cette protection ne joue pas en cas de modification de ces régimes. Dans ce cas, les modifications adoptées devront être conformes aux règles constitutionnelles. La question se pose donc de savoir ce qu'elles imposent en matière de financement public des cultes et si les règles de financement peuvent varier selon les territoires. 


\subsection{LA QUESTION DE LA CONFORMITÉ DE RÉGIMES TERRITORIAUX DE FINANCEMENT PUBLIC DES CULTES AVEC LES PRINCIPES CONSTITUTIONNELS}

Si les régimes territoriaux de financement public des cultes existants ne sont pas inconstitutionnels en raison de la volonté du constituant de les préserver, on peut cependant s'interroger sur leur compatibilité avec l'interprétation donnée par le Conseil constitutionnel de plusieurs principes constitutionnels.

\subsubsection{Le principe de laïcité}

La compatibilité de l'existence de financements publics des cultes avec le principe de laïcité ne paraît pas contestable dans sa globalité. Ce ne sont que les modalités de ce financement qui font discussion, et tout d'abord la possibilité même d'un financement public des cultes.

Ainsi que cela a déjà été évoqué, il est désormais admis que le principe constitutionnel de laïcité inscrit à l'article 2 de la Constitution n'exclut pas tout financement des cultes. Cette analyse déjà formulée depuis plusieurs années par la doctrine ${ }^{31}$ a été confirmée par le Conseil d'État ${ }^{32}$ et consacrée par le Conseil constitutionnel ${ }^{33}$. Ce dernier n'a pas donné valeur constitutionnelle à la disposition de l'article 2 de la loi de 1905 selon laquelle « la République [...] ne subventionne aucun culte ». Au demeurant, en dehors même du cas particulier de certains territoires, ce principe fait l'objet de nombreuses exceptions législatives ${ }^{34}$ qui deviendraient inconstitutionnelles si le non-financement était consacré comme une règle générale au plan constitutionnel ${ }^{35}$. Une interdiction constitutionnelle absolue de financement public des cultes n'est donc guère concevable. Pour autant, tout financement public des cultes n'est pas compatible avec le principe constitutionnel de laïcité.

Le Conseil constitutionnel a rappelé plusieurs limitations au financement public des cultes imposées par le principe de laïcité : la neutralité de l'État, la «non-reconnaissance » des cultes, le respect de toutes les croyances, l'égalité

31. Woehrling J.-M., « Réflexions sur le principe de la neutralité de l'État en matière religieuse et sa mise en œuvre en droit français », ASSR, n 101, 1998, p. 31-52.

32. CE, 16 mars 2005, précit.

33. Cons. const., déc. 21 févr. 2013, précit.

34. V. Messner F., Prélot P.-H., Woehrling J.-M. (dir.), Traité de droit français des religions, Paris, LexisNexis , $2^{\mathrm{e}}$ éd. 2013, p. 1395 et s.

35. Woehrling J.-M., "L'interdiction pour l'État de financer ou de reconnaître un culte: quelle valeur juridique? », RDP 2006, p. 1633. 
de tous les citoyens devant la loi sans distinction de religion, le libre exercice des cultes, l'absence de salariat des cultes.

Ce dernier élément s'oppose au régime particulier existant en AlsaceMoselle et en Guyane qui comporte une rémunération directe par une personne publique des ministres du culte. Il s'agit d'un aspect non accessoire de ces régimes territoriaux, mais aussi non essentiel. En effet, la mise en place d'une dotation globale au bénéfice d'institutions religieuses à la place d'une rémunération directe des ecclésiastiques ne constituerait pas de problème majeur. On ne voit pas non plus en quoi elle ferait progresser de manière substantielle la neutralité de l'État. Par ailleurs, cette interprétation du principe constitutionnel de laïcité paraît aussi poser des problèmes au regard de la rémunération des aumôniers en droit commun dans les prisons, dans les hôpitaux et dans l'armée.

Au total, le principe de laïcité ne constitue pas en lui-même un obstacle à une diversification territoriale des modalités de financement public des cultes, dès lors qu'au regard de ce principe une pluralité de mises en œuvre est concevable, sans que l'une d'entre elles apparaisse nécessairement devoir s'imposer à toutes les autres et éliminer par voie de conséquence des variations territoriales. La question est plus complexe au regard du principe d'égalité.

\subsubsection{Le principe d'égalité}

Un traitement différencié selon les territoires de la question du financement public des cultes pourrait être regardé comme une atteinte à la règle de l'égalité des citoyens devant la loi. À cette interrogation, il faut répondre en plusieurs temps.

Il est tout d'abord désormais admis par la doctrine comme par la jurisprudence que le principe de l'unicité et de l'indivisibilité de la République n'implique pas l'uniformité législative. Le principe d'indivisibilité s'oppose à un démembrement interne de la République, mais non à l'existence de règles législatives propres à certaines parties du territoire. Il n'existe pas de principe constitutionnel d'unité législative, en vertu duquel la loi doit concerner de manière uniforme tout le territoire national. «L'indivisibilité de la République suppose un ordonnancement constitutionnel unique, mais peut s'accommoder d'un ordonnancement législatif non uniforme ${ }^{36}$. Elle signifie essentiellement, indivisibilité de la souveraineté et s'oppose à un démembrement interne de la

36. Flauss J.-F., « Droit local alsacien-mosellan et Constitution », RDP 1992, p. 1625. 
République, mais non à l'existence de règles législatives propres à certaines parties du territoire ${ }^{37}$.

Aussi, en vertu d'une jurisprudence constante du Conseil constitutionnel, il est loisible pour le législateur de définir des règles particulières pour certains territoires, s'il existe pour cela un motif d'intérêt général. Le Conseil constitutionnel a ainsi déclaré constitutionnelles les règles particulières applicables à l'Île-de-France ${ }^{38}$ ou instituant un statut particulier pour la Corse ${ }^{39}$. C'est dans une décision relative à l'aménagement du territoire que le Conseil constitutionnel a explicitement admis que le principe d'égalité pouvait prendre en compte la diversité des territoires ${ }^{40}$. De nombreuses dispositions législatives s'appliquent ainsi de manière différenciée au plan territorial. Le Conseil constitutionnel n'a censuré à ce jour aucune de ces adaptations législatives, dès lors que cette différentiation se fait sur des critères objectifs et pour des buts rationnels, tenant compte de la différence de situation.

Pour les collectivités d'outre-mer, la possibilité de règles législatives particulières est expressément prévue par la Constitution. Celle-ci distingue cependant entre plusieurs situations : dans les départements et régions d'outre-mer (notamment la Guyane), si les lois et règlements sont applicables de plein droit, ils peuvent faire l'objet d'adaptations tenant aux caractéristiques de ces collectivités. Il appartient au législateur de justifier la nécessité de ces adaptations $^{41}$. La mesure ne doit toutefois pas aller au-delà de la simple adaptation. De plus, l'article 73 précise qu'elle ne doit pas porter sur les garanties des libertés publiques, dont fait partie la liberté des cultes. Pour les collectivités d'outre-mer (en particulier la Polynésie française et Saint-Pierre et Miquelon), l'article 74 de la Constitution prévoit un statut de "spécialité législative » qui tient compte des intérêts de chaque collectivité : les lois et règlements de droit commun n'y sont applicables que si cela est prévu expressément. Des règles particulières peuvent concerner des aspects très importants. Seules doivent

37. Lemaire F., Le principe d’indivisibilité de la République: mythe et réalité, Presses universitaires de Rennes, 2010.

38. Cons. const., déc. 29 déc. 1989, n 89-270 DC, Loi de finances rectificative pour 1989 ; déc. 6 mai 1991, n 91-291 DC, Loi instituant une dotation de solidarité urbaine.

39. Cons. const., déc. 25 févr. 1982, n 82-138 DC, Loi portant statut particulier de la région de Corse ; déc. 17 janv. 2002, n² 2001-454 DC, Loi relative à la Corse.

40. Cons. const., déc. 26 janv. 1995, n 94-358 DC, Loi d'orientation pour l'aménagement et le développement du territoire; V. MÉLIn-SOUCRAMANiEn F., « Les adaptations du principe d'égalité à la diversité des territoires ", RFDA 1997, p. 906.

41. Cons. const., déc. 25 juill. 1984, n 84-174 DC, Loi relative aux compétences des régions de Guadeloupe, de Guyane, de Martinique et de la Réunion; déc. 7 déc. 2000, n²000-435 DC, Loi d'orientation pour l'outre-mer. 
être appliquées uniformément dans les collectivités d'outre-mer, comme sur le reste du territoire, les lois dites de "souveraineté », telles que celles relatives à la défense nationale, à la nationalité et la capacité des personnes, au statut des agents de la fonction publique, aux droits des citoyens dans leurs relations avec l'État, etc. Enfin, pour la Nouvelle-Calédonie, les articles 76 et 77 de la Constitution prévoient une autonomie encore plus grande.

S'agissant de règles territoriales qui concernent le domaine des libertés publiques, des précisions complémentaires doivent être données : il convient de noter que dès lors qu'il le justifie par des intérêts propres ou par un motif d'intérêt général, le législateur peut aussi prendre des règles territoriales en matière de libertés publiques. Il existe une jurisprudence constitutionnelle restreignant les variations dans la mise en œuvre de libertés publiques, mais elle concerne les collectivités territoriales ${ }^{42}$. Le principe de l'uniformité de l'exercice des droits et libertés fondamentaux ${ }^{43}$ ne s'applique qu'aux collectivités décentralisées et non au législateur national. On sait, par exemple, que l'exercice du droit de suffrage connaît des conditions variables selon les territoires ${ }^{44}$. Par ailleurs, la règle dégagée par le Conseil constitutionnel selon laquelle le principe de l'unicité du peuple français « s'oppose à ce que soient reconnus des droits collectifs à quelque groupe que ce soit, défini par une communauté d'origine, de culture, de langue ou de croyance ${ }^{45}$ ne concerne pas les territoires, mais des groupes susceptibles de se distinguer du peuple français. La distinction territoriale relève du droit objectif. Elle ne se confond pas avec l'attribution de droits subjectifs particuliers à des personnes ou à des groupes. Il en est de même du principe d'égalité entre collectivités territoriales $^{46}$ : celui-ci ne s'oppose pas à la création de statuts particuliers ${ }^{47}$, ni a des

42. Une telle limitation du pouvoir législatif serait manifestement trop restrictive au regard de la marge d'action que la Constitution laisse à ce dernier et compte tenu de la nécessité de prendre en compte les spécificités notamment territoriales. Quoi qu'il en soit, cette question ne met pas en cause le pouvoir du législateur de faire varier au plan territorial les éléments non essentiels des libertés fondamentales.

43. Cons. const., déc. 9 avr. 1996, n 96-373 DC, Loi organique portant statut d'autonomie de la Polynésie française.

44. V. les règles particulières aux villes de Paris, Lyon, Marseille par exemple.

45. Cons. const., déc. 15 juin 1999, n 99-412 DC, Charte européenne des langues régionales ou minoritaires.

46. Cons. const., déc. 3 juill . 1986, n 86-209 DC, Loi de finances rectificative pour 1986.

47. Cons. const., déc. 9 mai 1991, n 91-290 DC, Loi portant statut de la collectivité territoriale de Corse : le législateur peut créer une catégorie de collectivité territoriale composée d'un seul élément. 
régimes propres à certaines collectivités territoriales, mais il permet de vérifier que ces régimes spécifiques disposent d'une justification suffisante ${ }^{48}$.

Il résulte de ce qui précède que, pour les collectivités d'outre-mer de l'article 74 de la Constitution et la Nouvelle-Calédonie, l'existence de règles particulières dans le domaine du financement des cultes ne devrait pas susciter d'objections constitutionnelles, puisque la Constitution elle-même écarte le principe d'égalité en ce qui les concerne. Pour les autres territoires, la question est de savoir, d'une part, si le financement des cultes doit être regardé comme concernant l'exercice d'une liberté publique et, d'autre part, s'il existe une « situation particulière » pour le territoire concerné justifiant l'existence des dispositions territoriales spécifiques en matière de financement des religions. Une réponse certaine n'est pas possible, mais une position restrictive du Conseil constitutionnel est probable.

\subsection{LES CONSÉQUENCES POUR L'ÉVOLUTION FUTURE DE RÉGIMES TERRITORIAUX DE FINANCEMENT PUBLIC DES CULTES}

Il résulte des développements qui précèdent que la conservation des régimes territoriaux « historiques » de financement public des cultes ne se heurte pas à des contraintes constitutionnelles. Par contre, leur développement ou toute modification se traduisant par un éloignement du droit commun pourrait être contestée comme étant non conforme à la Constitution.

En matière de droit local alsacien-mosellan, cette limite ressort clairement de la décision Somodia du Conseil constitutionnel ${ }^{49}$. Celui a estimé « qu'à défaut de leur abrogation ou de leur harmonisation avec le droit commun, ces dispositions particulières ne peuvent être aménagées que dans la mesure où les différences de traitement qui en résultent ne sont pas accrues et que leur champ d'application n'est pas élargi ». Le Conseil constitutionnel semble ainsi poser une règle d'immobilisme au droit local : à défaut de s'aligner sur le droit général, celui-ci doit rester en l'état ! La formulation de cette règle est très générale. Il faut considérer cependant qu'elle ne s'applique que pour

48. Ainsi dans la décision $n^{\circ}$ 2009-588 DC du 6 août 2009 (Loi réaffirmant le principe du repos dominical), le Conseil constitutionnel a estimé au sujet de Paris, au regard de l'objet du nouvel article L. 3132-25 du Code du travail, c'est-à-dire la procédure de classement d'une commune ou d'une zone touristique, qu' « aucune différence de situation ne justifie que le pouvoir de proposition, qui appartient dans la législation en vigueur au conseil de Paris, ne soit pas confié au maire de Paris comme dans l'ensemble des autres communes, y compris Lyon et Marseille».

49. Cons. const., déc. 5 août 2011, n²011-157 QPC, RFDA 2012, p. 131, note WoEHrLing J.-M. 
autant que la disposition locale nouvelle heurte un principe constitutionnel. En fait, le Conseil constitutionnel l'applique non seulement à une modification du droit local historique, mais aussi, en vertu du principe d'égalité, à toute disposition nouvelle concernant l'Alsace et la Moselle ${ }^{50}$, refusant ainsi de reconnaître l'existence dans ces trois départements d'une situation particulière justifiant une législation spécifique, même nouvelle.

Si l'on applique le même raisonnement aux autres régimes territoriaux particuliers en matière de financement public des cultes, on devrait en conclure que ceux-ci sont condamnés eux aussi soit à la disparition, soit à la « cristallisation ».

Cela est d'autant plus paradoxal qu'on reproche parfois aux régimes territoriaux particuliers leur immobilisme et de ne pas avoir évolué pour mieux prendre en compte les nouveaux cultes. Ainsi, en Alsace-Moselle, on fait grief au système des « cultes statutaires » de ne pas faire une place au " culte musulman ». Mais en même temps, on explique que le cadre constitutionnel s'oppose à toute évolution de la « liste » des « cultes statutaires».

Le renforcement des normes constitutionnelles ne joue donc pas en faveur d'une reconnaissance de la diversité territoriale pour gérer le fait religieux, notamment en matière de financement public, mais s'exerce plutôt dans le sens d'une uniformisation renforcée.

Au début des années 2000, le système institutionnel français semblait s'ouvrir à une certaine diversification territoriale des normes, y compris en matière de gestion du fait religieux : interprétation souple du principe de laïcité, développement des régimes d'autonomie en outre-mer, décentralisation et régionalisation en métropole, intérêt positif pour le modèle du droit local, recours à l'expérimentation, compréhension souple de la notion d'égalité, etc. Ces différentes potentialités ne se sont finalement pas réalisées. Le cadre actuel semble revenir à une vision plus traditionnelle de l'unité/uniformité. Il est marqué par un double aspect : non remise en cause pour des motifs constitutionnels des régimes historiques, mais aussi enfermement de ceux-ci dans un cadre non évolutif qui les condamne à terme.

50. Cons. const., déc. 26 sept. 2014, n²014-414 QPC, Sté Assurances du Crédit Mutuel. 